\title{
Ranking relations between diseases, drugs and genes for a curation task
}

\author{
Simon Clematide ${ }^{*}$, Fabio Rinaldi
}

From Machine Learning for Biomedical Literature Analysis and Text Retrieval in the International Conference on Machine Learning and Applications 2011

Honolulu, HI, USA. 18-21 December 2011

* Correspondence: simon. clematide@uzh.ch

Institute of Computational Linguistics, University of Zurich, Binzmühlestrasse 14, 8050 Zurich, Switzerland

\begin{abstract}
Background: One of the key pieces of information which biomedical text mining systems are expected to extract from the literature are interactions among different types of biomedical entities (proteins, genes, diseases, drugs, etc.). Several large resources of curated relations between biomedical entities are currently available, such as the Pharmacogenomics Knowledge Base (PharmGKB) or the Comparative Toxicogenomics Database (CTD).

Biomedical text mining systems, and in particular those which deal with the extraction of relationships among entities, could make better use of the wealth of already curated material.

Results: We propose a simple and effective method based on logistic regression (also known as maximum entropy modeling) for an optimized ranking of relation candidates utilizing curated abstracts. Furthermore, we examine the effects and difficulties of using widely available metadata (i.e. MeSH terms and chemical substance index terms) for relation extraction. Cross-validation experiments result in an improvement of the ranking quality in terms of AUCiP/R by 39\% (PharmGKB) and $116 \%$ (CTD) against a frequency-based baseline of 0.39 (PharmGKB) and 0.21 (CTD). For the TAP-10 metrics, we achieve an improvement of 53\% (PharmGKB) and 134\% (CTD) against the same baseline system (0.21 PharmGKB and 0.15 CTD).

Conclusions: Our experiments with the PharmGKB and the CTD database show a strong positive effect for the ranking of relation candidates utilizing the vast amount of curated relations covered by currently available knowledge databases. The tasks of concept identification and candidate relation generation profit from the adaptation to previously curated material. This presents an effective and practical method suitable for conservative extension and re-validation of biomedical relations from texts that has been successfully used for curation experiments with the PharmGKB and CTD database.
\end{abstract}

\section{Biomed Central}

(c) 2012 Clematide and Rinaldi; licensee BioMed Central Ltd. This is an open access article distributed under the terms of the Creative Commons Attribution License (http://creativecommons.org/licenses/by/2.0), which permits unrestricted use, distribution, and reproduction in any medium, provided the original work is properly cited. 


\section{Background}

The wealth of published information in the biomedical domain is at the same time an opportunity and a challenge. Accessing this information, and making sense of it, becomes an increasingly difficult task which requires a considerable expertise. In order to help the biologists quickly locate the essential information that they need, different organizations provide curated databases, which organize the available knowledge about a particular specific subject, for example UniProt/SwissProt [1] is one of the most authoritative resources concerning proteins, BioGrid [2] is the broadest database describing gene and protein interactions. Most reference databases are created and maintained using a very costly and expensive manual curation procedure, which involves highly skilled professionals. It has been observed already a few years ago that such an approach is not sufficiently efficient in order to cope with the increasing quantity of published results [3]. In order to support this process, researchers are turning their attention to text mining methodologies, not with the aim of replacing manual curation, which we consider not possible in the foreseeable future, but rather with the aim of providing tools that can make the curation process more efficient. Clearly such tools will need to be tailored to the specific task or database where they are going to be deployed, however some major tendencies are already clear and will shape the future development of the field. Some of the fundamental tasks that text mining systems are required to deal with are: term recognition, entity identification and the detection of important relations between entities.

The text mining community has been organizing a number of shared tasks aiming at providing an infrastructure for the comparative evaluation of different text mining technologies. One such task, which is of particular relevance to the work described in this paper, is the protein-protein interaction task which took place in the 2006 and 2009 editions of the BioCreative competitive evaluations $[4,5]$. The organizers provide a collection of annotated documents as a training dataset (typically derived from one of the curated databases) and a separate collection of unannotated documents as a test dataset. Participants have a limited time frame to process the training data and deliver results back to the organizers, who will then score these results against a previously withheld gold standard, using a set of metrics suited to the task. In this paper we focus on a different type of relations, namely those among genes, drugs/chemicals and diseases, and we use information derived from the PharmGKB database [6,7] and the CTD database [8] as our gold standard. These gold standards could be used in a text mining task analogous to the proteinprotein interaction task defined in the BioCreative competitions.

We propose and evaluate a simple and practical method to achieve a high-quality ranked list of candidate relations based on the output of a term recognizer. Once entities have been identified, candidate relations can be generated with simple techniques, for example, co-occurrence within the same text span. However, such candidates would be too numerous to be useful, so proper ranking techniques are necessary in order to render these results accessible and really useful for a curation task. We use a machine learning approach suited for reranking of candidate relations by applying a maximum entropy method that integrates information from the vast amount of already curated relations from the PharmGKB and the CTD. This paper concludes with a brief overview of an integrated curation environment where the results described in the paper are applied. 


\section{Methods}

First we give a proper characterization of the resources and the gold standard data derived from the PharmGKB and the CTD databases. Next we present the evaluation measures and tools used for the experiments. Then we continue to describe our methods for term recognition, entity scoring, relation extraction and relation candidate ranking.

\section{Resources}

In order to perform simple and replicable experiments we refrain from more sophisticated and resource-intensive entity recognition approaches and do not use any external database of names and identifiers, for instance, by leveraging synonyms from the UMLS [9] or BioPortal [10]. Instead we restrict the terminological dictionaries to the ones provided by the PharmGKB resp. the CTD that can be downloaded in a plain textual format. These resources include terms used in the curated papers and their unique identifiers for each corresponding entity. For the PharmGKB, we have 30351 terms (2986 IDs) for drugs, 28633 terms (3198 IDs) for diseases, 176366 terms (28633 IDs) for genes. For the CTD, we have 388384 terms (101030 IDs) for chemicals, 69483 terms (9657 IDs) for diseases, 711631 terms (79837 IDs) for genes. The terms for chemicals and disease of the CTD are largely from $\mathrm{MeSH}$. The relationship data as available from the databases are represented as binary combination between two typed identifiers, supplemented with additional information regarding the type of evidence supporting the relationship. For all experiments described in this paper, we limit the set of relations to the ones based upon manually curated evidence from PubMed. In particular, we do not use inferred relations from the CTD and automatically created relation annotations from the PharmGKB, which were accessible in the past through their web interface.

From the PharmGKB, we get 26122 binary relations, which are based upon 5062 distinct PubMed articles. However, the number of relations attributed to an article varies strongly between just 1 up to 600 relations per article. Given that we consider only abstracts and not full-text, the task of extracting more than two dozens of relations seems not realistic. We therefore decided to restrict the data set for our experiments to all articles containing at most 20 relations. The resulting 4658 articles, which we then used for our experiments, contain 14825 relations. The source databases include some reflexive relations, i.e. relations between identical concepts, which we removed from our dataset. Table 1 shows the exact distribution of all relation types in our experimental data set split up by the number of relations in an article. As can be seen there, relations between the three different entity types, i.e. diseases (henceforth "Di"), drugs ("Dr") and genes ("Ge"), do not occur uniformly. In our data set, about $42 \%$ of all relations are of type Drug-Gene (Dr-Ge), about $37 \%$ of type Disease-Gene (Di-Ge) and only $18 \%$ of type Disease-Drug (Di-Dr). Relations between entities of the same type do exist, but they are marginal and contribute only about $3 \%$ of all relations.

From the CTD, we get 294151 binary relations, which are based upon 27960 distinct PubMed articles. However, the number of relations attributed to an article varies strongly between just 1 up to more than 9500 relations per article. Given the fact that the CTD provides a lot more gold standard relations we restrict the data set for our experiments with the CTD to all articles containing at most 12 relations. The remaining 23257 articles contain 71856 relations. The lower part of Table 1 shows the exact distribution for these relations for the CTD. In this data set, about 73\% are of type Chemical-Gene (for the sake 
Table 1 Distribution of relations per article in experimental data sets.

\begin{tabular}{|c|c|c|c|c|c|c|c|c|c|c|c|c|c|}
\hline \multicolumn{14}{|c|}{ PharmGKB data set } \\
\hline \multirow{2}{*}{$\begin{array}{r}\text { \#Rels } \\
\text { per Art }\end{array}$} & \multicolumn{2}{|c|}{ Di-Di } & \multicolumn{2}{|c|}{ Di-Dr } & \multicolumn{2}{|c|}{ Di-Ge } & \multicolumn{2}{|c|}{ Dr-Dr } & \multicolumn{2}{|c|}{ Dr-Ge } & \multicolumn{2}{|c|}{ Ge-Ge } & \multirow{2}{*}{$\begin{array}{r}\text { all } \\
\text { sum } \\
\end{array}$} \\
\hline & abs & rel & abs & rel & abs & rel & abs & rel & abs & rel & abs & rel & \\
\hline 1 & 2 & 0.1 & 129 & 6.6 & 842 & 43.0 & 29 & 1.5 & 938 & 47.9 & 19 & 1.0 & 1959 \\
\hline 2 & 6 & 0.5 & 138 & 10.9 & 484 & 38.1 & 18 & 1.4 & 611 & 48.1 & 13 & 1.0 & 1270 \\
\hline 3 & 1 & 0.0 & 705 & 26.5 & 925 & 34.8 & 12 & 0.5 & 993 & 37.4 & 22 & 0.8 & 2658 \\
\hline 4 & 9 & 1.2 & 98 & 13.1 & 231 & 30.9 & 21 & 2.8 & 372 & 49.7 & 17 & 2.3 & 748 \\
\hline 5 & 0 & 0.0 & 397 & 24.3 & 575 & 35.2 & 15 & 0.9 & 636 & 38.9 & 12 & 0.7 & 1635 \\
\hline 6 & 7 & 1.1 & 62 & 9.6 & 237 & 36.6 & 19 & 2.9 & 301 & 46.5 & 22 & 3.4 & 648 \\
\hline 7 & 1 & 0.1 & 154 & 20.0 & 293 & 38.1 & 6 & 0.8 & 296 & 38.4 & 20 & 2.6 & 770 \\
\hline 8 & 0 & 0.0 & 155 & 18.5 & 334 & 39.8 & 17 & 2.0 & 320 & 38.1 & 14 & 1.7 & 840 \\
\hline 9 & 12 & 1.6 & 153 & 19.8 & 283 & 36.6 & 12 & 1.6 & 279 & 36.0 & 35 & 4.5 & 774 \\
\hline 10 & 10 & 4.2 & 32 & 13.3 & 74 & 30.8 & 0 & 0.0 & 114 & 47.5 & 10 & 4.2 & 240 \\
\hline 11 & 1 & 0.1 & 205 & 28.2 & 236 & 32.5 & 4 & 0.6 & 270 & 37.2 & 10 & 1.4 & 726 \\
\hline 12 & 12 & 3.4 & 67 & 19.3 & 87 & 25.0 & 11 & 3.2 & 165 & 47.4 & 6 & 1.7 & 348 \\
\hline 13 & 0 & 0.0 & 47 & 18.1 & 100 & 38.5 & 6 & 2.3 & 107 & 41.2 & 0 & 0.0 & 260 \\
\hline 14 & 0 & 0.0 & 52 & 19.5 & 118 & 44.4 & 0 & 0.0 & 93 & 35.0 & 3 & 1.1 & 266 \\
\hline 15 & 7 & 1.7 & 77 & 18.3 & 144 & 34.3 & 0 & 0.0 & 189 & 45.0 & 3 & 0.7 & 420 \\
\hline 16 & 0 & 0.0 & 40 & 19.2 & 100 & 48.1 & 0 & 0.0 & 68 & 32.7 & 0 & 0.0 & 208 \\
\hline 17 & 0 & 0.0 & 39 & 17.6 & 51 & 23.1 & 7 & 3.2 & 106 & 48.0 & 18 & 8.1 & 221 \\
\hline 18 & 0 & 0.0 & 2 & 1.2 & 56 & 34.6 & 0 & 0.0 & 100 & 61.7 & 4 & 2.5 & 162 \\
\hline 19 & 0 & 0.0 & 36 & 23.7 & 59 & 38.8 & 0 & 0.0 & 57 & 37.5 & 0 & 0.0 & 152 \\
\hline 20 & 0 & 0.0 & 127 & 24.4 & 203 & 39.0 & 4 & 0.8 & 166 & 31.9 & 20 & 3.8 & 520 \\
\hline TOTAL & 68 & 0.5 & 2715 & 18.3 & 5432 & 36.6 & 181 & 1.2 & 6181 & 41.7 & 248 & 1.7 & 14825 \\
\hline \multicolumn{14}{|c|}{ CTD data set } \\
\hline \#Rels & & & \multicolumn{2}{|c|}{ Di-Dr } & \multicolumn{2}{|c|}{ Di-Ge } & \multicolumn{4}{|c|}{ Dr-Ge } & & & all \\
\hline per Art & & & abs & rel & abs & rel & & & abs & rel & & & sum \\
\hline 1 & & & 1482 & 23.3 & 1333 & 21.0 & & & 3539 & 55.7 & & & 6354 \\
\hline 2 & & & 2454 & 21.9 & 1539 & 13.7 & & & 7219 & 64.4 & & & 11212 \\
\hline 3 & & & 1806 & 17.6 & 1154 & 11.3 & & & 7294 & 71.1 & & & 10254 \\
\hline 4 & & & 1717 & 15.5 & 994 & 9.0 & & & 8357 & 75.5 & & & 11068 \\
\hline 5 & & & 1144 & 15.3 & 507 & 6.8 & & & 5824 & 77.9 & & & 7475 \\
\hline 6 & & & 1248 & 14.5 & 525 & 6.1 & & & 6855 & 79.5 & & & 8628 \\
\hline 7 & & & 578 & 12.7 & 270 & 6.0 & & & 3688 & 81.3 & & & 4536 \\
\hline 8 & & & 648 & 14.1 & 225 & 4.9 & & & 3719 & 81.0 & & & 4592 \\
\hline 9 & & & 396 & 14.4 & 165 & 6.0 & & & 2184 & 79.6 & & & 2745 \\
\hline 10 & & & 285 & 12.7 & 88 & 3.9 & & & 1867 & 83.3 & & & 2240 \\
\hline 11 & & & 193 & 13.7 & 93 & 6.6 & & & 1122 & 79.7 & & & 1408 \\
\hline 12 & & & 203 & 15.1 & 63 & 4.7 & & & 1078 & 80.2 & & & 1344 \\
\hline TOTAL & & & 12154 & 16.9 & 6956 & 9.7 & & & 52746 & 73.4 & & & 71856 \\
\hline
\end{tabular}

This table shows how many relations between which entities occur per article in both data sets. PharmGKB has some relations between entities of the same type. CTD contains only relations between entities of different types. In order to keep the tables of both databases easily comparable, entities of type "chemical" from CTD are labeled with "Dr" (drugs).

of comparability with the PharmGKB, we recast the CTD entity type "chemical" as "Dr"), about $17 \%$ are of type Disease-Chemical (Di-Dr), and only $10 \%$ are of type Disease-Gene (Di-Ge). The CTD contains no relations between entities of the same type.

\section{Measures and tools for evaluation}

The format of the relationship file provided by the knowledge bases lends itself to an easy transformation into a format equivalent to the one used for the protein-protein 
interaction (PPI) task of BioCreative II.5 [5]. Given a text mining tool which can produce a ranked list of gene/drug/disease relations, it becomes then possible to score these results against the knowledge base data by using a scoring tool provided by the BioCreative organizers.

The BioCreative PPI evaluation tool returns results according to the standard metrics used in information retrieval (Precision, Recall, F-Measure) as well as a more novel measure called "AUC iP/R" (area under the curve of the interpolated precision/recall graph). The AUC iP/R measure (not to be confused with the more frequently used "AUC of the ROC curve" metric) provides an indication of the quality of the ranking of the candidate relations. The intuitive idea is that, given equivalent $P / R / F$ figures, correct predictions which occur towards the top of the ranked list are more useful than the ones which are lower in the ranking. The implicit assumption is that a curator could use the ranking to decide where to stop looking at the candidate results, therefore a better ranking provides a better user experience. The AUC iP/R curve is defined in [11], a detailed operative description of AUC iP/R, as used in the BioCreative evaluations, can be found at http://www.biocreative.org/tasks/biocreative-ii5/biocreative-ii5-evaluation/.

A recently proposed alternative evaluation measure for ranked results is the "Threshold Average Precision" (TAP-k) [12], which (in slightly simplified terms) averages precision for the results above a given error threshold. The TAP-k metric is easier to interpret and also directly relevant for the end user, who in most cases would not be willing to inspect a long list of candidate relations containing many false positives. The TAP-k mirrors the fact that a curator will stop validating a list of ranked relation candidates after having rejected a certain number $k$ of false positives. In our main experiments, we set $k=10$.

Note, that the values of the evaluation metrics reported here are always macro-averaged, i.e. the mean of the evaluation score is computed separately for each article.

\section{Text processing and term recognition}

For the experiments we use PubMed abstracts corresponding to the PubMed IDs mentioned by the relationship files from the knowledge bases. It would of course be desirable to work on full papers rather than abstracts, however, not all these publications are freely accessible, and most importantly, they are not available in a common format. The lack of a common format hinders the usability of full-text publications for practical text mining purposes, as it makes it more difficult to identify significant parts of the papers (e.g. results sections) or distinguish elements that require special processing (e.g. tables).

In the experiments, we apply the first processing steps of our OntoGene relation mining system (OG-RM) in order to annotate the input documents with the terminology provided by the respective knowledge bases. First, in the preprocessing stage, the PubMed XML is transformed into a custom XML format where sentences and tokens boundaries are identified using the LingPipe framework (for more information see http://alias-i.com/lingpipe). Second, the OntoGene pipeline proceeds with a step of term annotation [13,14]. In order to account for possible surface variants and in order to allow for partial matches, a normalization step is included in the annotation procedure. The annotations generated by the OntoGene pipeline can then be used to generate candidate relations using a number of different criteria. Since each token in the OntoGene annotation framework is assigned a unique identifier, extracted terms can be related back to their position in the text. 


\section{Selecting textual material and metadata}

The experimental settings described below vary according to the amount of metadata that is included in the text mining process:

1. only title and abstract of the article are used (henceforth $t$ );

2. additionally to $t$ the names in the chemical substance list of an abstract are used (henceforth tc);

3. additionally to $t$ the $\mathrm{MeSH}$ descriptors and their qualifiers are used (henceforth tm);

4. all possible information is used (henceforth tmc).

The motivation for the inclusion of metadata such as $\mathrm{MeSH}$ or chemical substance lists is an improved recall of the term recognition. Table 2 shows the exact improvement for our experimental data set from the PharmGKB. Diseases have the lowest coverage of $67 \%$ and profit, as expected, substantially from the inclusion of MeSH terms (+8\%). Drug recognition improves using the list of chemical substances $(+3 \%)$, but does not further improve by adding the MeSH terms. As our term recognizer is tuned towards the detection of proteins and genes, we reach the highest coverage for genes, as expected. Using the metadata still gives an improvement of $2 \%$. For all entities we cover $74 \%$ with text only $(t)$ and $78 \%$ using all metadata (tmc).

Table 2 Coverage of term recognition for concepts and relations in experimental data

\begin{tabular}{|c|c|c|c|c|c|c|c|c|c|}
\hline \multicolumn{10}{|c|}{ PharmGKB data set } \\
\hline \multirow[b]{2}{*}{ Entity } & \multirow[b]{2}{*}{$\mathbf{N}$} & \multicolumn{2}{|c|}{$t$} & \multicolumn{2}{|c|}{ tm } & \multicolumn{2}{|c|}{ tc } & \multicolumn{2}{|c|}{ tmc } \\
\hline & & abs & rel & abs & rel & abs & rel & abs & rel \\
\hline $\mathrm{Di}$ & 3830 & 2550 & 66.58 & 2872 & 74.99 & 2557 & 66.76 & 2872 & 74.99 \\
\hline Dr & 4751 & 3527 & 74.24 & 3632 & 76.45 & 3668 & 77.20 & 3668 & 77.20 \\
\hline $\mathrm{Ge}$ & 7522 & 5838 & 77.61 & 5930 & 78.84 & 5989 & 79.62 & 5994 & 79.69 \\
\hline TOTAL & 16103 & 11915 & 73.99 & 12434 & 77.22 & 12214 & 75.85 & 12534 & 77.84 \\
\hline Di-Di & 68 & 22 & 32.35 & 24 & 35.29 & 22 & 32.35 & 24 & 35.29 \\
\hline Di-Dr & 2715 & 1279 & 47.11 & 1484 & 54.66 & 1326 & 48.84 & 1494 & 55.03 \\
\hline Di-Ge & 5432 & 3102 & 57.11 & 3555 & 65.45 & 3181 & 58.56 & 3585 & 66.00 \\
\hline Dr-Dr & 181 & 128 & 70.72 & 132 & 72.93 & 135 & 74.59 & 135 & 74.59 \\
\hline Dr-Ge & 6181 & 3858 & 62.42 & 4016 & 64.97 & 4097 & 66.28 & 4099 & 66.32 \\
\hline Ge-Ge & 248 & 141 & 56.85 & 142 & 57.26 & 145 & 58.47 & 145 & 58.47 \\
\hline TOTAL & 14825 & 8530 & 57.54 & 9353 & 63.09 & 8906 & 60.07 & 9482 & 63.96 \\
\hline \multicolumn{10}{|c|}{ CTD data set } \\
\hline & & \multicolumn{2}{|c|}{$\mathbf{t}$} & \multicolumn{2}{|c|}{$\mathrm{tm}$} & \multicolumn{2}{|c|}{ tc } & \multicolumn{2}{|c|}{ tmc } \\
\hline Entity & $\mathbf{N}$ & abs & rel & abs & rel & abs & rel & abs & rel \\
\hline Di & 12639 & 6939 & 54.90 & 9502 & 75.18 & 6941 & 54.92 & 9502 & 75.18 \\
\hline $\mathrm{Dr}$ & 38523 & 27541 & 71.49 & 29531 & 76.66 & 30119 & 78.18 & 30129 & 78.21 \\
\hline Ge & 39150 & 28389 & 72.51 & 28975 & 74.01 & 29169 & 74.51 & 29199 & 74.58 \\
\hline TOTAL & 90312 & 62869 & 69.61 & 68008 & 75.30 & 66229 & 73.33 & 68830 & 76.21 \\
\hline Di-Ge & 6956 & 4117 & 59.19 & 5100 & 73.32 & 4163 & 59.85 & 5126 & 73.69 \\
\hline Dr-Di & 12154 & 5335 & 43.90 & 8219 & 67.62 & 5700 & 46.90 & 8356 & 68.75 \\
\hline Dr-Ge & 52746 & 31015 & 58.80 & 33971 & 64.40 & 34832 & 66.04 & 34883 & 66.13 \\
\hline TOTAL & 71856 & 40467 & 56.32 & 47290 & 65.81 & 44695 & 62.20 & 48365 & 67.31 \\
\hline
\end{tabular}

Distribution of identifiable gold standard concepts and relations given the output from our term recognizer and split according to the inclusion of metadata: text only ( $t$ ), text and MeSH terms (tm), text and chemical substance list (tc), text and all metadata $(\mathrm{tmc})$. 
The lower half of Table 2 shows the corresponding numbers for the CTD data. Diseases again have the lowest coverage for text only (55\%) and profit heavily from $\mathrm{MeSH}$ terms $(+20 \%)$. Chemical coverage improves mostly by information from the chemical substance list $(+7 \%)$, but the MeSH terms add also most of the important information. Detection of genes based on the text only is again high and improves slightly $(+2 \%)$ when metadata is added, but remains clearly beyond the recognition rate achieved for the PharmGKB. Regarding the coverage of relations, we see an improvement of $6 \%$ in the case of the PharmGKB, and almost $11 \%$ for the CTD.

\section{Relation extraction and relation ranking}

There are several ways in which the entities recognized in an abstract can be combined, for example by co-occurrence in the same sentence, or by using a set of syntactic filters as done in our previous work on protein-protein interactions $[15,16]$. The approach which delivers the maximal recall is to generate all pairwise undirected combinations of all entities identified in the abstract.

As shown in Table 2 for the PharmGKB, this approach can deliver a recall of $58 \%$ using only text ( $\mathrm{t}$ ), $63 \%$ using additionally $\mathrm{MeSH}(\mathrm{tm})$ and $64 \%$ using all the metadata (tmc). Note, that the upper limit of the recognition rate varies strongly by the type of entities involved in a relation, disease-drug relations have an unexpected low upper limit in PharmGKB. The lower part of Table 2 shows similar numbers for the CTD, i.e., a recall of 56\% ( $\mathrm{t}$ ), 66\% using MeSH (tm) and 67\% using chemical substance lists as well (tmc). Considering that only abstracts were used, this seems a reasonable term recognition coverage for our experiments. However, this approach will massively overgenerate, therefore ranking of the results becomes absolutely necessary.

In order to reduce the overgeneration of relation candidates, one could limit the set of candidate relations to entities that co-occur at least once in the same sentence. However, experiments we performed with such co-occurrence limits resulted in inferior performance. Table 3 explains this rather unexpected effect to some degree: for about $30 \%$ of the relations from the gold standard where our term recognizer is able to detect both entities in the article there is no sentence containing a hit for both entities in the PharmGKB. For the CTD, about $32 \%$ of the gold standard relation cannot be found in the same sentence. A term recognizer with improved acronym detection and coreference resolution may alleviate this problem.

\section{Ranking relations by frequency}

A baseline ranking of all candidate relations of an abstract can be generated on the basis of the number of occurrences of the respective entities:

$$
\text { relscore }\left(e_{1}, e_{2}\right)=\frac{f\left(e_{1}\right)+f\left(e_{2}\right)}{f(E)}
$$

where $f\left(e_{1}\right)$ and $f\left(e_{2}\right)$ are the number of times the entities $e_{1}$ and $e_{2}$ are observed in the abstract, while $f(E)$ is the total count of all entities in the abstract. Once a score is assigned to each candidate pair, it is possible to filter out the most unlikely candidates, either by setting a threshold value for the score, or by selecting only the N-best candidates. Using one of these filtering techniques will result into variable values of Precision, Recall and FMeasure, depending on the exact value of the score threshold, or $\mathrm{N}$ parameter. 
Table 3 Occurrence of gold standard relations in the same sentence

\begin{tabular}{rrrrrr}
\hline \multicolumn{5}{c}{ PharmGKB data set } \\
\hline In Same Sentence & \multicolumn{1}{c}{ In Diff. Sentences } \\
Relation & Absolute & Relative & & Relative & Total \\
\hline Dr-Dr & 111 & 86.7 & 17 & 13.3 & 128 \\
Ge-Ge & 113 & 80.1 & 28 & 19.9 & 141 \\
Dr-Ge & 2895 & 75.0 & 963 & 25.0 & 3858 \\
Di-Ge & 2009 & 64.8 & 1093 & 35.2 & 3102 \\
Di-Dr & 816 & 63.8 & 463 & 36.2 & 1279 \\
Di-Di & 13 & 59.1 & 9 & 40.9 & 22 \\
\hline All & 5957 & 69.8 & 2573 & 30.2 & 8530 \\
\hline \multicolumn{7}{c}{ CTD data set } & & & \\
\hline Relation & In Same Sentence & In Diff. Sentences & Relative & Total \\
\hline Di-Ge & Absolute & Relative & & 24.4 & 4575 \\
Dr-Ge & 23457 & 75.6 & 1118 & 33.0 & 34488 \\
Dr-Di & 3948 & 67.0 & 11365 & 33.4 & 5930 \\
\hline All & 30528 & 67.9 & 14465 & 32.1 & 44993 \\
\hline
\end{tabular}

Distribution of all gold standard relations where both entities could be identified by our term recognizer. An occurrence of a relation is categorized as "In Same Sentence" if there exists at least one sentence in a given abstract where both entities co-occur. An occurrence of a relation is categorized as "In Different Sentences" if both entities can be found in a given abstract but never co-occur together in the same sentence. For these tables metadata such as MeSH terms and chemical substance lists were not included.

\section{Title occurrence boosting}

We know from our previous experiments [15] that giving a "boost" to the entities contained in the title can produce a measurable improvement of ranking of the results (measured by the AUC or TAP metrics). We have empirically verified that a sensible boost for abstracts is around 10. This is equivalent to counting the entities in the title ten times. In the rest of this paper, boosted frequencies of entities are expressed as $f_{b}(e)$.

The baseline approach for relation ranking described above will be referred to as $\mathrm{m} 0$ in the rest of this paper.

\section{Preferring relations between unequal types}

As shown in Table 1, relations between entities of the same type occur far less often in the PharmGKB than relations between different types. We can model this empirical fact by applying a type preference coefficient to the relation score that affects relations between entities of the same type. An empirically set coefficient of $1 / 10$ proved to be useful:

$$
\text { typepref }\left(e_{1}, e_{2}\right)= \begin{cases}0.1 & \text { if both entities have the same type } \\ 1 & \text { otherwise. }\end{cases}
$$

In the experiments described in Section 'Results and discussion', we express the application of the type coefficient in the following way:

1. no type preference applied (henceforth e0);

2. type preference applied (henceforth e1).

Additionally, we experimented with using the relative frequency of a relation type taken from the training set as a type preference coefficient. Because results deteriorated 
consistently when using this setting, we do not take it into account for the evaluation. Note that the CTD does not contain relations between equal types, therefore the experimental settings for the CTD do not vary this parameter.

\section{Scoring entities for being part of curated gold standard relations}

The ranking of relation candidates using a simple frequency-based confidence score derived from textual evidence can be further optimized if we apply a supervised machine learning method (in our case a Maximum Entropy technique) that models the relevance of an entity using the curated relations from the gold standard and the documents where these relations occur. In our experiments described below, results computed using this technique will be tagged as $\mathrm{m} 1$.

There are two motivations for scoring concepts with regard to relation ranking: First, we want to identify automatically the false positive entities that our term recognizer detects in order to penalize them. The term recognizer eagerly modifies term entries from the dictionary while matching, i.e. material is removed from an entry in the term dictionary in order to allow for partial matches, or on-the-fly acronyms are created. For instance, the term form "neuronal" may be identified as the genes PA134898200, PA134924203, PA134896732 from the term database because they have "neuronal protein" as one of their lexical entries. Once identified such false positive partial matches could be ruled out by ad-hoc rules. However, for different terminological resources different rules may be necessary. We regard a general approach that works independently from the used terminological resources and that achieves an automatic adaptation as highly beneficial. In order to deal with such cases, we need not only to condition on the entities, but also on their textual representation.

Second, we need to adapt to highly ranked false positive relations which are generated by our frequency based approach by frequent but irrelevant entities. The goal is to identify some global (dis)preference that can be found in the PharmGKB or the CTD relationships.

\section{Normalizing term forms}

For a precise description of the ME-optimized ranking approach, we need to introduce some notation. In the following, the notation $t$ refers to a normalized textual form of a recognized term. In the experiments, we vary four levels of normalization:

1. no normalization except lower-case initial characters (henceforth $\mathrm{n} 0$ );

2. lower-case characters and some punctuation removed: $’ \backslash \backslash() \backslash /$ - (henceforth $\mathrm{n} 1$ );

3. lower-case characters and only alphanumeric characters retained in tokens (henceforth n2);

4. same as 3, but token boundaries are removed (henceforth $\mathrm{n} 3$ ).

For instance, "FC ( gamma ) - receptor" is normalized to "fC gamma receptor" in mode $\mathrm{n} 1$, in mode $\mathrm{n} 3$ we get "fcgammareceptor". Multiple spaces resulting from the deletion of characters are squeezed into one. [17] have shown that the removal of punctuation symbols does not harm the term recognition quality. The combination of a term $t$ and one of its valid entities $e$ is noted as $t: e$.

\section{Applying counting caps}

Because term frequency in an article seems crucial for an estimation of the relevance of a concept, we condition valid term-entity combinations additionally on their number of occurrences in an article. In order to reduce the resulting problem of data 
sparseness we apply different upper limits (so-called caps) on the raw frequencies:

$$
f^{c}(t: e)= \begin{cases}c a p & \text { if } f(t: e) \geq c a p \\ f(t: e) & \text { otherwise }\end{cases}
$$

In the experiments, we test different settings:

1. cap $=\infty$, i.e no cap is used (henceforth $\mathrm{c} 0$ );

2. $c a p=1$, i.e. a term-entity is present or not (henceforth $\mathrm{c} 1$ );

3. $c a p=3$, cap $=6$, cap $=9$ (henceforth $\mathrm{c} 3, \mathrm{c} 6, \mathrm{c} 9$ ).

\section{Estimating gold probabilities}

Next we define a predicate $\operatorname{gold}(A, e)$ which is true (i.e. 1$)$ for an article $A$ if there is at least one relation in the gold standard where entity $e$ is part of, and false (i.e. 0 ) otherwise. Using the notions defined beforehand, we specify the overall probability of an entity $e$ of being part of a gold relation given the entity $e$, a term form $t$, and their frequency $f^{c}(t: e)$ in article $A$ :

$$
P\left(\operatorname{gold}(A, e)=1 \mid e, t, f^{c}(t: e)\right)
$$

We estimate $P\left(\operatorname{gold}(A, e)=1 \mid e, t, f^{c}(t: e)\right)$ with the help of the Maximum Entropy Modeling tool megam [18] using the recognized terms of the abstracts from a training set together with the gold standard information from the same document set. Technically, each value $e, t, f^{c}(t: e)$ from an article serves as a joint feature for the maximum entropy classifier and the value of $\operatorname{gold}(A, e)$ as its binomial class, i.e. a number between 0 and 1 . This numeric value will be predicted by the model when features from unseen articles are presented. The model of a maximum entropy classifier consists of a weight for each feature of the training material. Formally, a conditional Maximum Entropy Model (aka. Logistic Regression) has the following exponential form:

$$
\begin{aligned}
p_{\lambda}(y \mid x) & =\frac{1}{Z(x)} \exp \left(\sum_{i} \lambda_{i} F_{i}(x, y)\right) \\
Z(x) & =\sum_{y} \exp \left(\sum_{i} \lambda_{i} F_{i}(x, y)\right),
\end{aligned}
$$

where $y$ is the joint feature $e, t, f^{c}(t: e)$ and $x$ is the value of the gold predicate $\operatorname{gold}(A, e)$. In the formula, we designate Maximum Entropy features by $F_{i}$ as the notation $f$ is used for frequencies in this paper. The Maximum Entropy Modeling tool iteratively optimizes the feature weights $\lambda$ in such a way that they maximize the conditional log-likelihood of the training material. There are two practical reasons for our choice for Maximum Entropy modeling: Firstly, this classifier does not suffer when dependent features are used, such as our smoothing features introduced below. Therefore, an approach as for instance a Naive Bayes classifier is not generally feasible for our method. Secondly, the Maximum Entropy tools performs very efficiently with ten thousands of features and it requires no parameter tuning as for example most Support Vector Machine tools.

\section{Smoothing counts}

For features not present in the training material there are no weights available. In order to reduce the resulting sparse data problem, we apply a smoothing method that works as follows: for each feature $e, t, f^{c}(t: e)$ add all additional features e, $t, n$ with $f^{c}(t: e)>n \geq 1$. In our 
experiments described in the section 'Results and discussion', we evaluate the effect of feature smoothing as follows:

1. do not smooth (henceforth s0);

2. apply smoothing (henceforth $\mathrm{s} 1$ ).

In the case of applying a cap of 1 (i.e. $\mathrm{c} 1$ ), smoothing (i.e. s1) is not necessary and the equation for the gold probability simplifies to the following:

$$
P(\operatorname{gold}(A, e=1 \mid t: e)
$$

For unseen terms $t$, i.e. terms not present in the training data, the maximum entropy classifier assigns a default probability based on the distribution of all training instances. However, we can specify better back-off probabilities if we take into account the admissible entity/entities $e$ of term $t$. Our current back-off model works as follows: if the entity $e$ of an unseen $t$ is seen in the article, the averaged probability of all seen term-entity pairs is used. Otherwise, the averaged probability of all entities of the same type as $e$ is used.

\section{Scoring entities}

Finally, the resulting score of an entity $e$ in an article $A$ is the sum of the boosted term frequency weighted by the gold probability:

$$
\operatorname{score}(e)=\sum_{t: e \in A} f_{b}(t: e) \times P\left(\operatorname{gold}(A, e)=1 \mid e, t, f^{c}(t: e)\right)
$$

\section{Scoring relations}

Having determined the score of each entity $e$, we add them to a relation score similar to the baseline method:

$$
\text { relscore }\left(e_{1}, e_{2}\right)=\left(\operatorname{score}\left(e_{1}\right)+\operatorname{score}\left(e_{2}\right)\right) \times \operatorname{typepref}\left(e_{1}, e_{2}\right)
$$

This simple relation score function has the disadvantage that a single entity score with a high value produces a high relation score even if the other entity has a very low entity score. As an alternative we use the harmonic mean of both entity scores in order to decrease the relation score of entity combinations with highly disparate entity scores.

$$
\text { relscore } e_{h}\left(e_{1}, e_{2}\right)=2 \times \frac{\operatorname{score}\left(e_{1}\right) \times \operatorname{score}\left(e_{2}\right)}{\operatorname{score}\left(e_{1}\right)+\operatorname{score}\left(e_{2}\right)} \times \operatorname{typepref}\left(e_{1}, e_{2}\right)
$$

In the evaluation we encode the different relation score metrics as follows:

1. simple sum of entity scores (henceforth $\mathrm{r} 0$ );

2. harmonic mean of entity scores (henceforth $r 1$ ).

\section{Experimental settings at a glance}

For the cross-validation experiments described in the next section, we vary the following settings:

- title and abstract ( $t)$, including MeSH ( $t m)$, including chemical substances ( $t c)$, including all metadata $(\mathrm{tmc})$;

- no type preference coefficient (e0); preference coefficient for unequal type (e1); 
- relation score as sum ( $r 0)$ or as harmonic mean ( $r 1)$ of entity score;

- baseline approach (no weighting of entities) (mo) vs. maximum entropy (ME) weighting (m1);

- normalization of term forms for ME: first letter in lower-case (n0), all characters in lower-case and some punctuation marks removed ( $\mathrm{n} 1$ ), lower-case alphanumeric characters with spaces (n2), lower-case alphanumeric characters without spaces (n3);

- caps for ME features: no cap, i.e. raw counts (c0), cap of 1, 3, 6 or 9 (cn);

- smoothing of ME features is off ( $\mathrm{s} 0)$ or on (s1).

Note that the settings $\mathrm{n}, \mathrm{C}$ and $\mathrm{s}$ are only meaningful for the ME approach. The baseline system as mentioned in the following section is identified by the settings $t$ $e 0-r 0-m 0-n 0-c 0-s 0$ or $t-e 0-r 0-m 0$ for short. The setting el is only applicable to the PharmGKB.

\section{Results and discussion}

In this section, we report on the systematic stratified 10-fold cross-validation evaluation using all different experimental settings mentioned in the preceding section. All numbers presented in this section are means of 10 different runs. Our data sets from the PharmGKB and the CTD were split into subsets stratified according to the number of relations per article. See Table 1 for the distribution of the frequency of relations per article. Note that we did not enforce a stratified distribution of different relation types in all subsets.

Taking into consideration all valid configurations of experimental settings leads to several hundred combinations to test for and to the same number of results to compare. For reasons of space we focus our presentation and discussion on the most important question to be answered by our results: which feature setting contributes how much performance increase to the baseline system or improvements thereof? We give a tabular overview of performance increase in terms of TAP-10 (Table 4) and AUCiP/R (Table 5) separately for the PharmGKB and the CTD.

These tables give a concise compilation of the following information:

The mean and standard deviation (noted as "sd") from the 10-fold cross-validation results of a given setting.

- The single experimental parameter setting that needs to be changed in order to achieve the highest performance increase. Only if no single parameter with better performance can be found, two parameters (or more) may be changed at once.

- The absolute $(\Delta \mathrm{abs})$ and relative $(\Delta \mathrm{rel})$ amount of performance improvement.

- The statistical significance of the improvement given as the $\mathrm{p}$ value of a Wilcoxon signed rank test for dependent pairs.

- An estimate of the minimal improvement expected in $95 \%$ of all cases, i.e. the lower limit of the $95 \%$ confidence interval $\left(\Delta \mathrm{CI}_{l}\right)$ taken from the Wilcoxon test.

- Finally, the relative performance improvement in comparison to the baseline $\left(\Delta \operatorname{rel}_{b s}\right)$.

The Wilcoxon signed rank test for dependent pairs is used to assess whether the improvement is significant or due to chance. The experimental setting of 10-fold cross 
Table 4 Evaluation of performance increase of TAP-10

\begin{tabular}{|c|c|c|c|c|c|c|c|c|}
\hline \multicolumn{9}{|c|}{ PharmGKB data set } \\
\hline setting & mean & sd & $\Delta$ & $\Delta$ abs & $\Delta$ rel & $p$ value & $\Delta \mathrm{Cl}_{l}$ & $\Delta$ rel $_{b s}$ \\
\hline t-e0-r0-m0-no-c0-s0 & 0.27 & 0.010 & & & & & & \\
\hline t-e1-r0-mo-no-c0-s0 & 0.32 & 0.010 & e1 & +0.042 & +15.3 & $9.8_{-4}$ & +0.039 & +15.3 \\
\hline t-e1-r0-m1-no-c0-s0 & 0.35 & 0.010 & $\mathrm{~m} 1$ & +0.030 & +9.6 & $9.8_{-4}$ & +0.028 & +26.4 \\
\hline t-e1-r0-m1-no-c1-s0 & 0.37 & 0.012 & c1 & +0.019 & +5.5 & $9.8_{-4}$ & +0.017 & +33.4 \\
\hline t-e1-r1-m1-n0-c1-s0 & 0.39 & 0.012 & $\mathrm{r} 1$ & +0.028 & +7.6 & $9.8_{-4}$ & +0.025 & +43.6 \\
\hline tmc-e1-r1-m1-n0-c1-s0 & 0.41 & 0.012 & tmc & +0.016 & +4.2 & $9.8_{-4}$ & +0.011 & +49.6 \\
\hline tmc-e1-r1-m1-n2-c1-s0 & 0.41 & 0.011 & $\mathrm{n} 2$ & +0.003 & +0.7 & $9.8_{-4}$ & +0.001 & +50.6 \\
\hline tmc-e1-r1-m1-n2-c6-s1 & 0.42 & 0.009 & c6-s1 & +0.005 & +1.1 & $9.8_{-4}$ & +0.002 & +52.3 \\
\hline tmc-e1-r1-m1-n3-c6-s1 & 0.42 & 0.009 & n3 & +0.002 & +0.5 & $2.9-3$ & +0.001 & +53.0 \\
\hline \multicolumn{9}{|c|}{ CTD data set } \\
\hline setting & mean & sd & $\Delta$ & $\Delta \mathrm{abs}$ & $\Delta$ rel & $p$ value & $\Delta \mathrm{Cl}_{l}$ & $\Delta$ rel $_{b s}$ \\
\hline t-ro-m0-no-c0-s0 & 0.15 & 0.006 & & & & & & \\
\hline t-ro-m1-no-c0-s0 & 0.22 & 0.004 & $\mathrm{~m} 1$ & +0.074 & +49.5 & $9.8_{-4}$ & +0.072 & +49.5 \\
\hline t-ro-m1-n0-c1-s0 & 0.28 & 0.007 & $\mathrm{cl}$ & +0.055 & +24.7 & $9.8_{-4}$ & +0.052 & +86.5 \\
\hline tmc-r0-m1-n0-c1-s0 & 0.31 & 0.021 & tmc & +0.029 & +10.2 & $2.0_{-3}$ & +0.030 & +105.6 \\
\hline tmc-r1-m1-n0-c1-s0 & 0.32 & 0.021 & r1 & +0.014 & +4.6 & $9.8_{-4}$ & +0.013 & +115.0 \\
\hline tmc-r1-m1-n3-c1-s0 & 0.34 & 0.005 & n3 & +0.019 & +5.8 & $2.0_{-3}$ & +0.010 & +127.5 \\
\hline tmc-r1-m1-n3-c3-s1 & 0.35 & 0.005 & c3-s1 & +0.010 & +3.0 & $9.8-4$ & $+0: 007$ & +134.4 \\
\hline
\end{tabular}

These tables show the amount of performance increase by exchanging one experimental parameter by another parameter (or a parameter combination, if no single parameter exchange increases the performance). From one row of the table to the following row we select the parameter(s) that gives the highest performance increase as measured by the mean TAP-10 from the cross validation runs. The absolute performance increase $(\Delta a b s)$ and the relative increase $(\Delta$ rel) between two adjacent rows are reported in the corresponding columns. A Wilcoxon signed rank test for dependent pairs is used to assess whether the improvement is significant or due to chance. The last column shows the relative increase compared to the baseline setting $\left(\Delta \mathrm{rel}_{b s}\right)$.

Table 5 Evaluation of performance increase of AUCiP/R

\begin{tabular}{|c|c|c|c|c|c|c|c|c|}
\hline \multicolumn{9}{|c|}{ PharmGKB data set } \\
\hline setting & mean & sd & $\Delta$ & $\Delta$ abs & $\Delta$ rel & $p$ value & $\Delta \mathrm{Cl}_{l}$ & $\Delta$ rel $_{b s}$ \\
\hline t-e0-r0-mo-no-c0-so & 0.39 & 0.015 & & & & & & \\
\hline t-e1-r0-mo-no-co-so & 0.44 & 0.012 & e1 & +0.048 & +12.1 & $9.8_{-4}$ & +0.044 & +12.1 \\
\hline t-e1-r0-m1-no-c0-s0 & 0.49 & 0.014 & $\mathrm{~m} 1$ & +0.044 & +10.0 & $9.8_{-4}$ & +0.042 & +23.2 \\
\hline t-e1-r0-m1-n0-c1-s0 & 0.51 & 0.017 & C1 & +0.027 & +5.5 & $9.8_{-4}$ & +0.023 & +30.0 \\
\hline tmc-e1-r0-m1-n0-c1-s0 & 0.53 & 0.015 & tmc & +0.018 & +3.4 & $9.8_{-4}$ & +0.013 & +34.5 \\
\hline tmc-e1-r1-m1-n0-c1-s0 & 0.54 & 0.015 & r1 & +0.012 & +2.3 & $9.8_{-4}$ & +0.010 & +37.6 \\
\hline tmc-e1-r1-m1-n2-c1-s0 & 0.55 & 0.015 & $\mathrm{n} 2$ & +0.003 & +0.6 & $9.8_{-4}$ & +0.002 & +38.4 \\
\hline tmc-e1-r1-m1-n2-c3-s1 & 0.55 & 0.013 & c3-s1 & +0.003 & +0.5 & $1.5_{-2}$ & +0.001 & +39.1 \\
\hline tmc-e1-r1-m1-n3-c0-s1 & 0.55 & 0.014 & $\mathrm{n} 3-\mathrm{co}$ & +0.002 & +0.4 & $1.4_{-2}$ & +0.000 & +39.7 \\
\hline \multicolumn{9}{|c|}{ CTD data set } \\
\hline setting & mean & sd & $\Delta$ & $\Delta$ abs & $\Delta$ rel & $p$ value & $\Delta \mathrm{Cl}_{l}$ & $\Delta$ rel $_{b s}$ \\
\hline t-ro-mo-no-c0-s0 & 0.21 & 0.008 & & & & & & \\
\hline t-ro-m1-no-c0-s0 & 0.30 & 0.006 & $\mathrm{~m} 1$ & +0.090 & +42.4 & $9.8_{-4}$ & +0.087 & +42.4 \\
\hline t-r0-m1-no-c1-s0 & 0.37 & 0.009 & C1 & +0.072 & +23.7 & $9.8_{-4}$ & +0.068 & +76.1 \\
\hline tmc-r0-m1-n0-c1-s0 & 0.41 & 0.026 & $\operatorname{tmc}$ & +0.040 & +10.6 & $2.0_{-3}$ & +0.042 & +94.9 \\
\hline tmc-r0-m1-n3-c1-s0 & 0.43 & 0.006 & n3 & +0.018 & +4.3 & $2.9_{-3}$ & +0.005 & +103.3 \\
\hline tmc-r1-m1-n3-c1-s0 & 0.45 & 0.007 & r1 & +0.015 & +3.5 & $9.8_{-4}$ & +0.013 & +110.4 \\
\hline tmc-r1-m1-n3-c3-s1 & 0.46 & 0.007 & c3-s1 & +0.012 & +2.8 & $9.8_{-4}$ & +0.009 & +116.2 \\
\hline
\end{tabular}

This table gives the corresponding overview of feature-wise performance increase as in Table 4. See Table 4 for a detailed explanation on the interpretation of the columns. 
validation leads to a small sample size and additionally the differences of means used for this kind of comparison are not always normally distributed in our data. In order to be able to apply the same significance test to all settings, such a non-parametric significance test is more appropriate than the parametric $t$-test. The $\mathrm{p}$ values and the non-parametric $95 \%$ confidence interval are exact values and not normal approximations (the test for improvement is one-sided and therefore only the lower limit of improvement is actually shown in the tables). We use the function wilcox.exact() from the library exactRankTests of the statistical software framework R. See the documentation for more technical details. Further discussion of the appropriateness of significance tests on results gained by cross-validation can be found in $[19,20]$.

Although the tables mentioned above iteratively answer the question which settings actually increase the system performance, we know nothing about the upper limit of ranking performance (given the results from our term recognizer). In order to assess the distance to this upper bound, we take the results of our best system and build a perfect ranking on top of it by pushing all true positives in front of all its false positives. The Figures 1 and 2 plot this information for varying cut-off limits: the lower limit of performance is given by the baseline $(t-e 0-\mathrm{m} 0)$, the upper limit is derived from our best setting for the respective metrics.

\section{Evaluation of relation ranking: TAP-10}

The evaluation metrics of TAP- $k$ is of utmost significance for our application scenario of database curation due to the fact that curators are not willing to sort out a large number of false positive relation candidates. For both data sets we take $k=10$, which means that after having seen 10 false positives no further results are taken into consideration.

The upper part of Table 4 shows the feature-specific performance increase of TAP-10 for the PharmGKB. The type preference coefficient e1 improves the baseline most, followed by the application of ME. Note that metadata $(t \mathrm{mc})$ improves results only modestly for the PharmGKB, in fact, using metadata without applying ME optimization performs worse than the baseline. A cap of 9 (c9) never results in the best increase for TAP-10 on the PharmGKB nor does it in any other ranking evaluation. However, applying a cap of 6 seems to be the best strategy for the PharmGKB. As the baseline for the PharmGKB is already well performing, the overall relative improvement is limited to $53 \%$.

As shown for the CTD in the lower part of Table 4, the order of features leading to the highest performance is similar to the one from the PharmGKB. However, the addition of metadata has a much stronger impact for the CTD. One reason for that may be the use of $\mathrm{MeSH}$ terminology in the CTD dictionaries. Having frequency counts in the gold probability features (i.e. having a setting other than $\mathrm{C} 1$ ) leads to a relatively small performance increase. The best settings for the PharmGKB and the CTD only differ in the cap (c6 vs. c3), which supports the conclusion that the techniques are generally applicable. In the case of the CTD, the rather low baseline performance is improved by more than $134 \%$.

The plots in Figure 1 show that the best setting for the PharmGKB not only performs better in terms of absolute TAP scores than the best setting for the CTD. Additionally, the best setting from the PharmGKB reduces the distance to the upper limit far more than the best setting for the CTD. One possible explanation for this fact may be given by the different distribution of articles containing a single relation: in the PharmGKB 


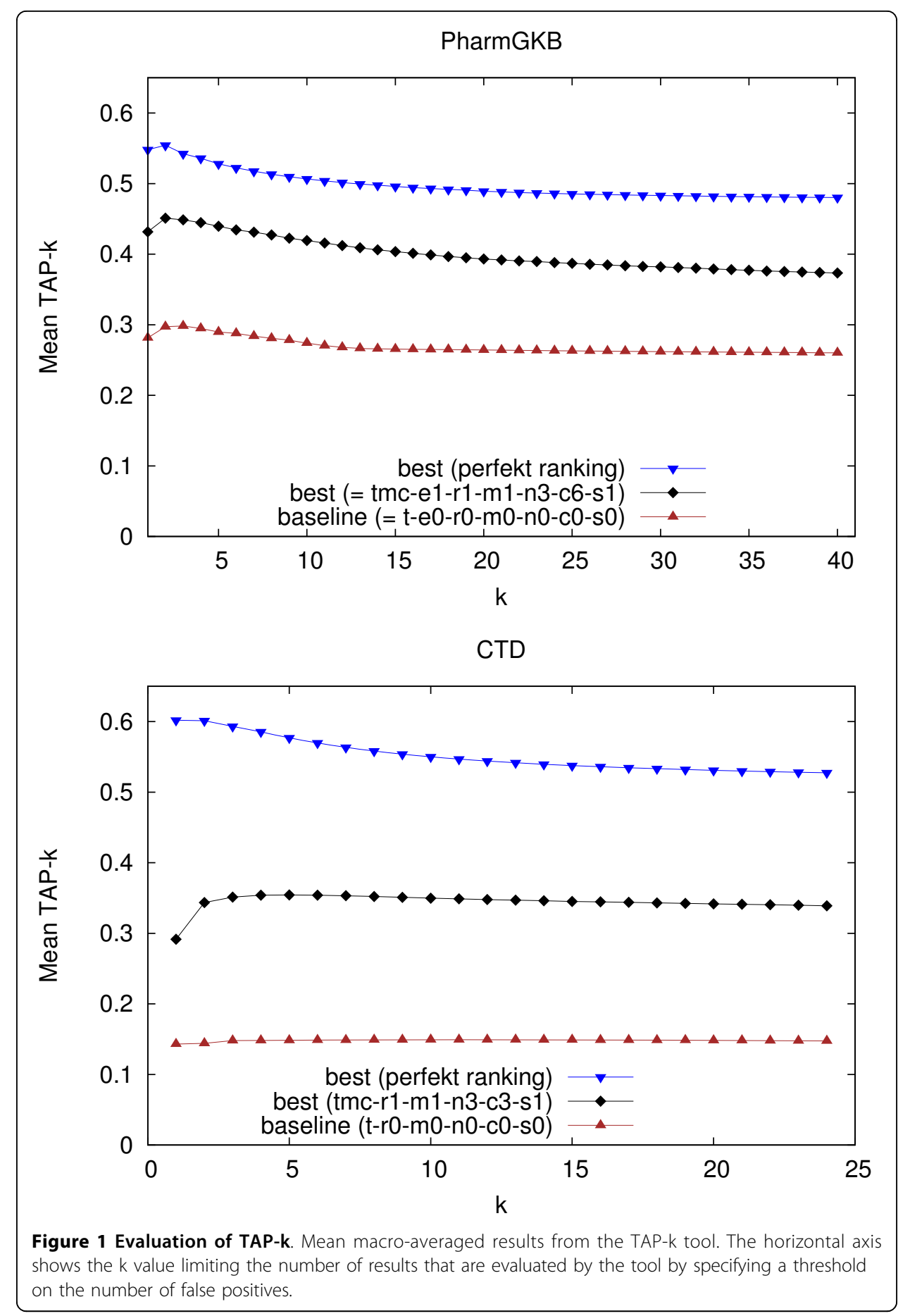

almost $40 \%$ of all articles contribute just one relation whereas in the CTD only about $22 \%$ do this.

\section{Evaluation of relation ranking: AUCiP/R}

According to our application scenario we apply a cut-off limit of 50 relations to all evaluations of AUCiP/R. The upper part of Table 5 shows the feature-specific performance increase for the PharmGKB. In contrast to the TAP measure, the addition of metadata is 


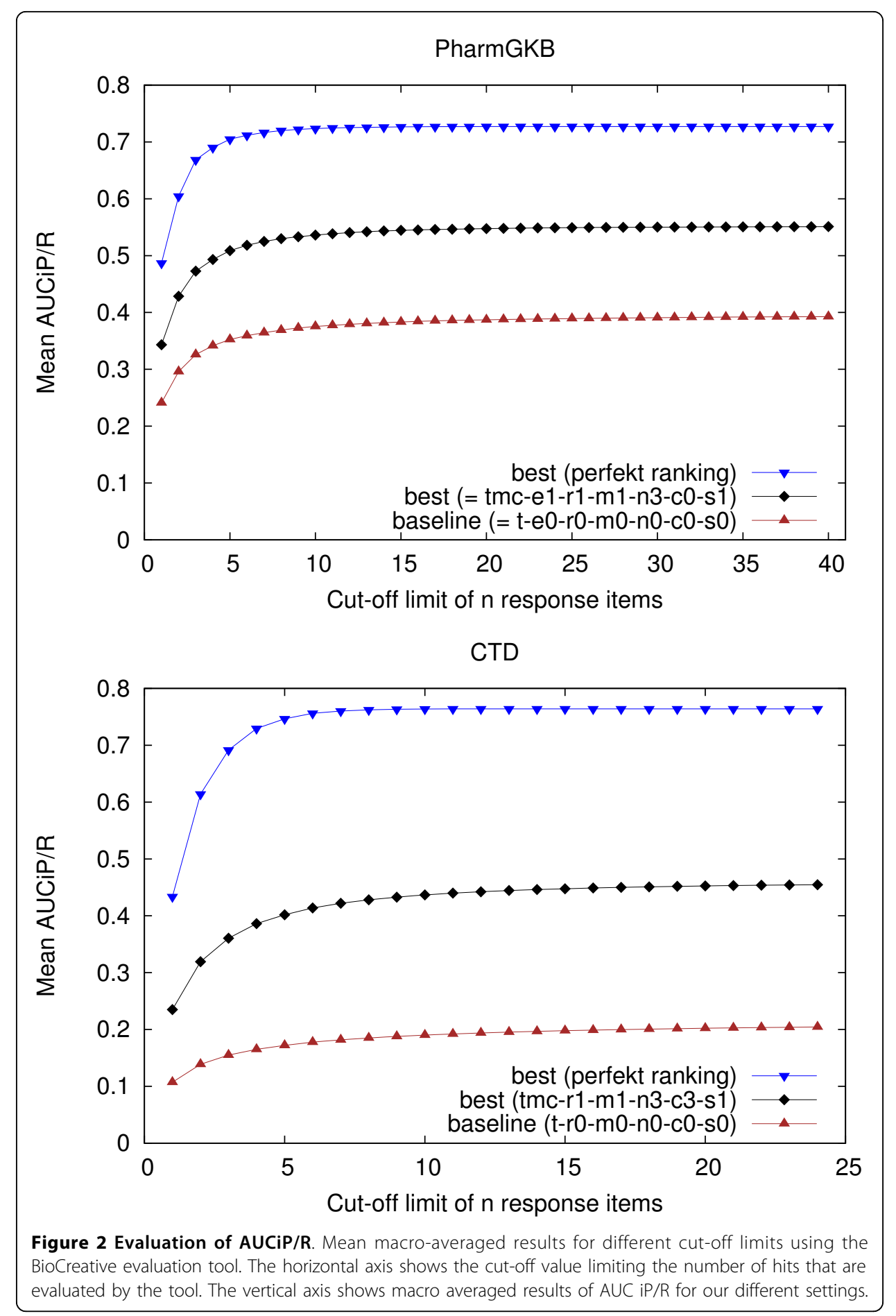

more important, thus expressing the fact that AUCiP/R is more sensitive to the improvement of recall than TAP- $k$. Again, determining the best settings for the CTD is more straightforward than for the PharmGKB. Although the improvement for the different performance increase steps are statistically significant, there are only small differences between the top settings. Note that the top setting for TAP-10 and for AUCiP/R are different for the PharmGKB. In contrast, the lower part of Table 5 shows almost the same 
feature ranking for the CTD for both evaluation metrics. We regard the switch between the order of $\mathrm{n} 3$ and $\mathrm{r} 1$ not as important, given the fact that the lower improvement confidence interval $\mathrm{CI}_{l}$ for $\mathrm{n} 3$ is much lower than the "random" empirical improvement of 0.0018. For the PharmGKB we achieve an overall improvement of $40 \%$. For the CTD, which again has a much lower baseline, the best setting improves by $116 \%$.

The plots in Figure 2 illustrate the dependency of AUCiP/R on recall. Note that whereas in Figure 1 the best settings for the PharmGKB seems apparently closer to the perfect ranking than the CTD, this difference is less prominent in terms of AUCiP/R.

\section{Evaluation of metadata contribution}

The inclusion of metadata such as MeSH or chemical substances into the text mining procedure improves the overall performance of relation ranking. Although this information is widely available from PubMed (or directly from the publishers), it may be missing for some texts. In Tables 6 we show how the performance of the best settings decreases from missing metadata. For the PharmGKB the difference is modest (under 5\%) if all metadata is discarded. For the CTD the TAP-10 score is almost 13\% higher if metadata is used. This difference correlates with the coverage improvements for metadata inclusion as shown in Table 2.

\section{Evaluation of Precision, Recall, and F-Measure}

The plots in Figure 3 show the corresponding numbers as computed by the Biocreative evaluation tool for the best system settings as resulting from the TAP-10 evaluation. Note that for small cut-offs, precision is high, e.g. the first solution is a correct relation in almost $60 \%$ of all cases on average in the PharmGKB, and almost 50\% in the CTD. However, precision drops quickly given the fact that there are not that many articles with more than 5 relations. For the PharmGKB the baseline performs better than the best system using cut-off limits $n>30$, which could be an adverse effect that our training material is limited to articles with at most 24 relations.

\section{Evaluation of the estimation of gold probabilities}

A substantial part of the performance of the maximum-entropy-based ranking depends on the proper estimation of the probability of an entity to be part of a true positive relation. Therefore, we evaluated the probability scores separately with regard to the experimental settings. Table 7 shows significant performance improvements by smoothing the feature counts ( $\mathrm{s} 1$ ), by using metadata ( $\mathrm{tmc}$ ), and by applying the strongest normalization (n3). Applying a cap of 9 (c9) improves minimally but is not statistically significant. Note that the best setting for the gold probability does not carry over as the best setting for TAP oder AUCiP/R.

\section{Usage in a curation environment}

Advanced text mining techniques are now reaching a maturity level that makes them increasingly relevant for the process of curation of biomedical literature. As part of our research in this area we developed a curation system called "OntoGene Document INspector" (ODIN [21]) which interfaces with our OntoGene text mining pipeline (OG-RM). We have used a version of ODIN for our participation to the 'interactive curation' task (IAT) of the BioCreative III competition [22]. This was an informal task without a quantitative 
Table 6 Evaluation of metadata contribution

\begin{tabular}{|c|c|c|c|c|c|c|c|c|}
\hline \multicolumn{9}{|c|}{ PharmGKB data set TAP-10 } \\
\hline setting & mean & sd & $\Delta$ & $\Delta$ abs & $\Delta$ rel & $p$ value & $\Delta \mathrm{Cl}_{I}$ & $\Delta \mathrm{rel}_{b s}$ \\
\hline t-e1-r1-m1-n3-c6-s1 & 0.40 & 0.011 & & & & & & \\
\hline tc-e1-r1-m1-n3-c6-s1 & 0.41 & 0.010 & tc & +0.006 & +1.4 & $9.8-4$ & +0.004 & +1.4 \\
\hline tm-e1-r1-m1-n3-c6-s1 & 0.41 & 0.011 & $\mathrm{tm}$ & +0.008 & +2.0 & $2.0_{-3}$ & +0.005 & +3.4 \\
\hline tmc-e1-r1-m1-n3-c6-s1 & 0.42 & 0.009 & tmc & +0.004 & +1.1 & $2.0_{-3}$ & +0.002 & +4.5 \\
\hline \multicolumn{9}{|c|}{ PharmGKB data set AUCiP/R } \\
\hline setting & mean & sd & $\Delta$ & $\Delta$ abs & $\Delta$ rel & $p$ value & $\Delta \mathrm{Cl}_{l}$ & $\Delta$ rel $_{b s}$ \\
\hline t-e1-r1-m1-n3-c0-s1 & 0.53 & 0.015 & & & & & & \\
\hline tc-e1-r1-m1-n3-c0-s1 & 0.53 & 0.014 & tc & +0.007 & +1.4 & $2.0_{-3}$ & +0.004 & +1.4 \\
\hline tm-e1-r1-m1-n3-c0-s1 & 0.54 & 0.015 & $\mathrm{tm}$ & +0.010 & +1.8 & $2.0_{-3}$ & +0.006 & +3.2 \\
\hline \multicolumn{9}{|c|}{ CTD data set TAP-10 } \\
\hline setting & mean & sd & $\Delta$ & $\Delta$ abs & $\Delta$ rel & $p$ value & $\Delta \mathrm{Cl}_{l}$ & $\Delta$ rel $_{b s}$ \\
\hline t-r1-m1-n3-c3-s1 & 0.31 & 0.006 & & & & & & \\
\hline tc-r1-m1-n3-c3-s1 & 0.33 & 0.005 & tc & +0.022 & +7.0 & $9.8-4$ & +0.019 & +7.0 \\
\hline tm-r1-m1-n3-c3-s1 & 0.34 & 0.004 & $\mathrm{tm}$ & +0.007 & +2.0 & $9.8-4$ & +0.005 & +9.2 \\
\hline tmc-r1-m1-n3-c3-s1 & 0.35 & 0.005 & $\operatorname{tmc}$ & +0.011 & +3.3 & $9.8_{-4}$ & +0.010 & +12.8 \\
\hline \multicolumn{9}{|c|}{ CTD data set AUCiP/R } \\
\hline setting & mean & sd & $\Delta$ & $\Delta$ abs & $\Delta$ rel & $p$ value & $\Delta \mathrm{Cl}_{I}$ & $\Delta$ rel $_{b s}$ \\
\hline t-r1-m1-n3-c3-s1 & 0.40 & 0.008 & & & & & & \\
\hline tc-r1-m1-n3-c3-s1 & 0.43 & 0.007 & tc & +0.030 & +7.5 & $9.8_{-4}$ & +0.027 & +7.5 \\
\hline tm-r1-m1-n3-c3-s1 & 0.45 & 0.006 & $\mathrm{tm}$ & +0.014 & +3.3 & $9.8-4$ & +0.012 & +11.1 \\
\hline tmc-r1-m1-n3-c3-s1 & 0.46 & 0.007 & $\operatorname{tmc}$ & +0.014 & +3.0 & $9.8_{-4}$ & +0.011 & +14.5 \\
\hline
\end{tabular}

This table shows the increase of performance for each step of inclusion of metadata as applied to best settings of TAP10 and AUCiP/R. See Table 4 for a detailed explanation on the interpretation of the columns.

evaluation of the participating systems. However, the curators who used the system commented positively on its usability for a practical curation task.

More recently, we have created a version of ODIN which allows inspection of abstracts automatically annotated with PharmGKB entities (the annotation is performed using OG-RM). Users can access either preprocessed documents, or enter any PubMed identifier and the corresponding abstract will be processed "on the fly". For the documents already contained in the PharmGKB it is also possible to compare the results of the system against the gold standard. The curator can inspect all entities annotated by the system, and easily modify them if needed (removing false positives with a simple click, or adding missed terms if necessary). The modified documents can be sent back for processing if desired, obtaining therefore modified candidate interactions. The user can also inspect the set of candidate interactions generated by the system, and act upon them just as on entities, i.e., confirm those which are correct, remove those which are incorrect. Candidate interactions are presented sorted according to the score which has been assigned to them by the text mining system, therefore the curator can choose to work with a small set of highly ranked candidates only, ignoring all the rest (see Figure 4). Recent user experiments using our curation environment, which makes use of the ranking proposed by the method described above, have shown positive results [23]. Additionally, a relation reranking on a CTD dataset, based on the approach described in this paper, has contributed to competitive results in the recent triage task (task 1) of the BioCreative 2012 shared task [24]. 


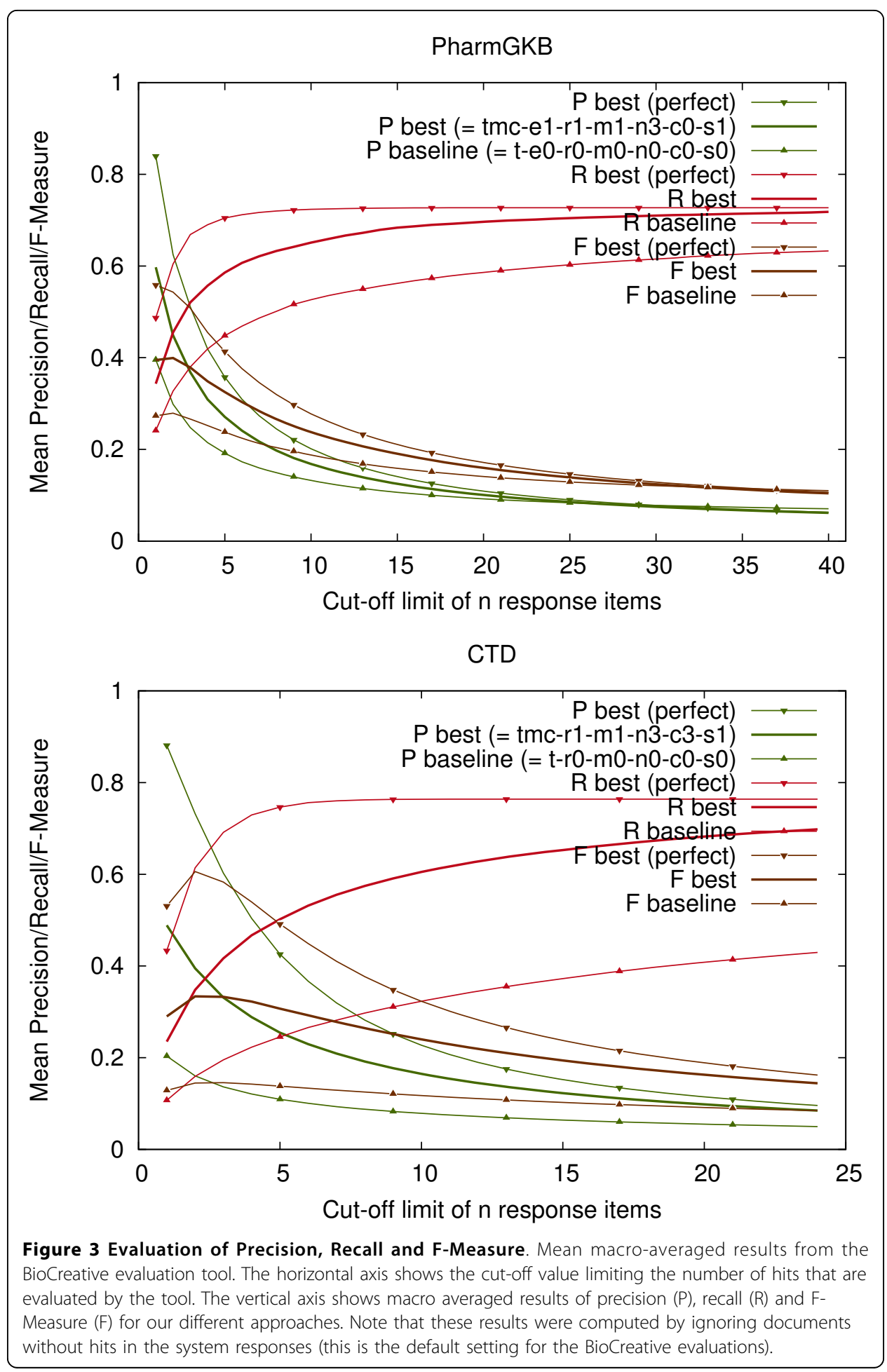

\section{Outlook}

As a continuation of this work, we would like to estimate the number of relations to be found in a paper on the basis of its textual content. Being able to provide this information before or at the initial stages of the curation process would help the curators to decide at which point of the curation process it is most sensible to stop after having found a given 
Table 7 Evaluation of performance increase of gold probability

\begin{tabular}{|c|c|c|c|c|c|c|c|c|}
\hline \multicolumn{9}{|c|}{ PharmGKB data set } \\
\hline setting & mean & sd & $\Delta$ & $\Delta$ abs & $\Delta$ rel & $p$ value & $\Delta \mathrm{Cl}_{l}$ & $\Delta$ rel $_{b s}$ \\
\hline $\mathrm{t}-\mathrm{m} 1-\mathrm{n} 0-\mathrm{co}-\mathrm{s} 0$ & 0.85 & 0.003 & & & & & & \\
\hline t-m1-n0-c0-s1 & 0.89 & 0.002 & s1 & +0.031 & +3.7 & $9.8-4$ & +0.030 & +3.7 \\
\hline tmc-m1-n0-c0-s1 & 0.90 & 0.002 & tmc & +0.016 & +1.8 & $9.8-4$ & +0.015 & +5.5 \\
\hline tmc-m1-n3-c0-s1 & 0.91 & 0.002 & n3 & +0.009 & +1.0 & $9.8-4$ & +0.008 & +6.5 \\
\hline tmc-m1-n3-c9-s1 & 0.91 & 0.002 & c9 & +0.000 & +0.0 & $3.5_{-1}$ & +0.000 & +6.5 \\
\hline \multicolumn{9}{|c|}{ CTD data set } \\
\hline setting & mean & sd & $\Delta$ & $\Delta$ abs & $\Delta$ rel & $p$ value & $\Delta \mathrm{Cl}_{I}$ & $\Delta$ rel $_{b s}$ \\
\hline t-m1-no-c0-s0 & 0.91 & 0.001 & & & & & & \\
\hline t-m1-n0-c0-s1 & 0.94 & 0.000 & s1 & +0.028 & +3.0 & $9.8-4$ & +0.027 & +3.0 \\
\hline t-m1-n3-c0-s1 & 0.95 & 0.001 & n3 & +0.010 & +1.1 & $9.8-4$ & +0.010 & +4.2 \\
\hline tm-m1-n3-c0-s1 & 0.95 & 0.001 & $\mathrm{tm}$ & +0.005 & +0.5 & $9.8-4$ & +0.005 & +4.7 \\
\hline tm-m1-n3-c6-s1 & 0.95 & 0.001 & c6 & +0.000 & +0.0 & $1.2_{-1}$ & +0.000 & +4.7 \\
\hline tmc-m1-n3-c6-s1 & 0.95 & 0.001 & tmc & +0.000 & +0.0 & $5.0_{-1}$ & +0.000 & +4.7 \\
\hline tmc-m1-n3-c9-s1 & 0.95 & 0.001 & C9 & +0.000 & +0.0 & $3.8_{-1}$ & +0.000 & +4.7 \\
\hline
\end{tabular}

This table gives the corresponding overview of feature-wise performance increase as Table 4. See Table 4 for a detailed explanation on the interpretation of the columns. The performance of the gold probability shows the quality of the Maximum Entropy approach for the estimation of an entity being part of a relation from the gold standard.

number of correct relations. This is particularly relevant because documents differ greatly in the number of relations they describe, ranging from a single relation to several hundred ones in a few documents describing high-throughput experiments. In the PharmGKB we have observed that $40 \%$ of the documents contain only one relation, however they contribute less than $10 \%$ of all relations. Approx. $90 \%$ of the documents contain 10 or less relations, however these documents contain around $50 \%$ of all relations. So the remaining $10 \%$ of documents (which contributes more than $50 \%$ of the relations) have a much higher number of relations per document. In the CTD $23 \%$ of the documents contain only one relation and contribute to $2.2 \%$ of all relations. Approx. $90 \%$ of the documents contain 12 or less relations.

A possible limitation of the proposed approach is that it favors conservative assumptions, i.e. it privileges entities and relationships which have already been seen over totally

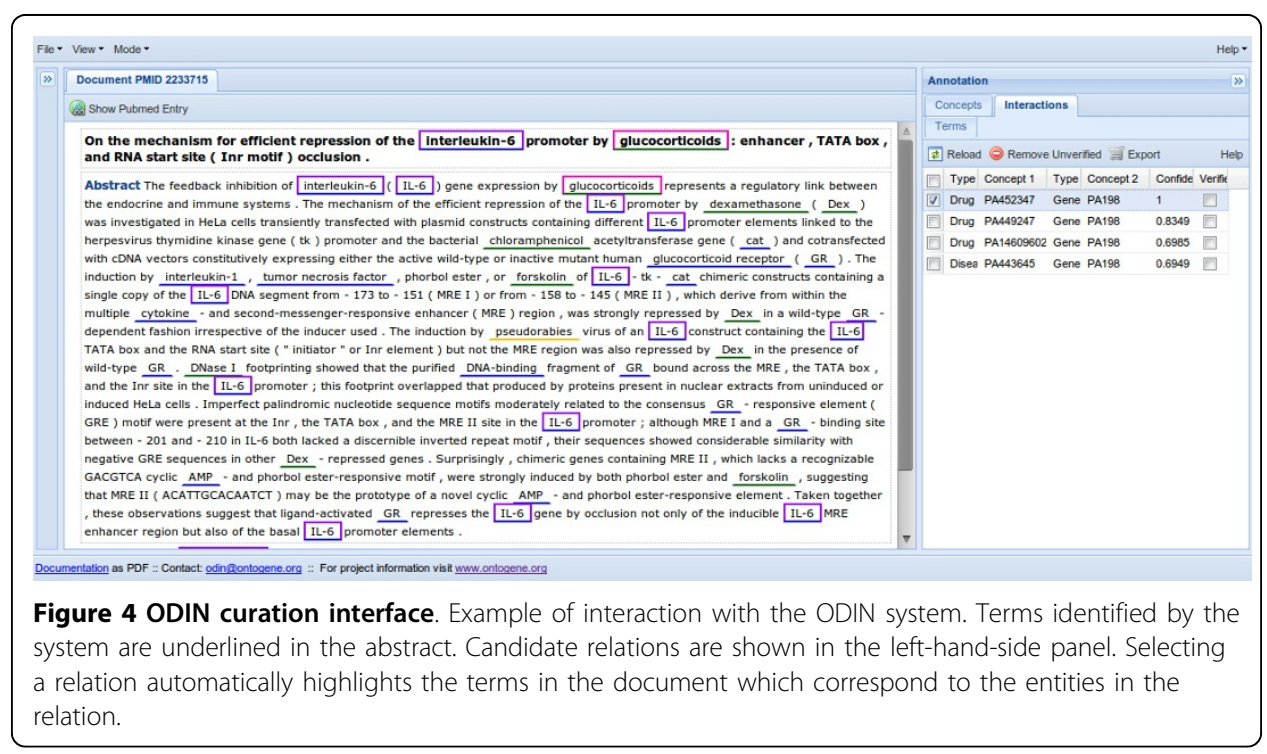


new entities and relationships. The inclusion of contextual and linguistic features might help compensate for this bias. A further question left for future work concerns the use and impact of alternative term recognizers (e.g. BANNER [25], MetaMap [26]) and additional terminological resources $[9,10]$.

\section{Conclusions}

We have presented a simple and practical approach for the mining and ranking of pharmacogenomic and toxicogenomic relations, and evaluated this approach systematically against two different knowledge bases, the PharmGKB and the CTD. We have implemented a Maximum Entropy technique for the optimized ranking of candidate relations using a purely frequency-based text mining approach. In order to estimate the relevance of a relation candidate for a new article, we combine textual evidence from the article with the evidence derived from the large set of relations found in curated articles. Our experiments show that this approach is feasible, and our results might offer a useful baseline for further developments that apply more sophisticated techniques from the field of protein-protein interaction detection [27]. Whereas for the experiments described in this paper we use only simple frequencybased features, the next step is to include contextual [28,29] and linguistic [30] features. The Maximum Entropy technique we applied so far is ideally suited for doing this.

We have used existing tools to score the results and to provide reliable evaluation metrics, including not only the traditional Precision, Recall and F-Measure, but also the increasingly important measures of ranking quality, such as AUC iP/R or TAP-k. The evaluation shows that the reranking techniques described in this article bring a considerable improvement to the results.

Finally, we have briefly mentioned the usage of these results within an assisted curation environment (ODIN), which is discussed more extensively in separate publications $[23,24]$. The experience from these experiments suggests that the usability of a curation environment is enhanced considerably by the presentation of properly ranked relation candidates.

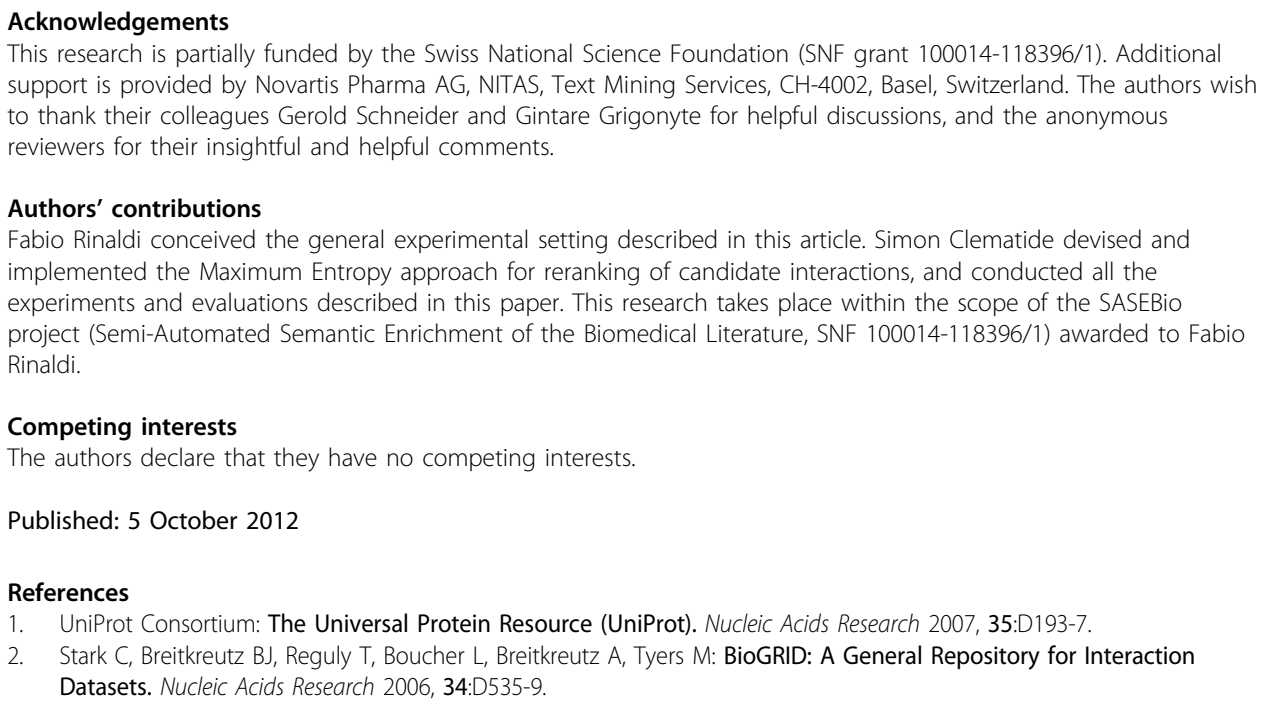


3. Baumgartner WA, Cohen KB, Fox LM, Acquaah-Mensah G, Hunter L: Manual curation is not sufficient for annotation of genomic databases. Bioinformatics 2007, 23(13):i41-48 [http://bioinformatics.oxfordjournals.org/cgi/content/abstract/23/ 13/i41].

4. Krallinger $M$, Leitner $F$, Rodriguez-Penagos $C$, Valencia A: Overview of the protein-protein interaction annotation extraction task of BioCreative II. Genome Biology 2008, 9(Suppl 2):S4.

5. Leitner F, Mardis SA, Krallinger M, Cesareni G, Hirschman LA, Valencia A: An Overview of BioCreative II.5. IEEE/ACM Transactions on Computational Biology and Bioinformatics 2010, 7(3):385-399.

6. Klein T, Chang J, Cho M, Easton K, Fergerson R, Hewett M, Lin Z, Liu Y, Liu S, Oliver D, Rubin D, Shafa F, Stuart J, Altman R: Integrating Genotype and Phenotype Information: An Overview of the PharmGKB Project. The Pharmacogenomics Journal 2001, 1:167-170.

7. Sangkuhl K, Berlin DS, Altman RB, Klein TE: PharmGKB: Understanding the Effects of Individual Genetic Variants. Drug Metabolism Reviews 2008, 40(4):539-551 [http://informahealthcare.com/doi/abs/10.1080/03602530802413338].

8. Davis A, King B, Mockus S, Murphy C, Saraceni-Richards C, Rosenstein M, Wiegers T, Mattingly C: The Comparative Toxicogenomics Database: update 2011. Nucleic Acids Res 2011.

9. Lindberg C: The Unified Medical Language System (UMLS) of the National Library of Medicine. J Am Med Rec Assoc 1990, 61(5):40-42.

10. Noy NF, Shah NH, Whetzel PL, Dai B, Dorf M, Griffith N, Jonquet C, Rubin DL, Storey MA, Chute CG, Musen MA: BioPortal: ontologies and integrated data resources at the click of a mouse. Nucleic Acids Research 2009, 37(suppl 2): W170-W173 [http://nar.oxfordjournals.org/content/37/suppl2/W170.abstract].

11. Manning CD, Raghavan P, Schütze H: Introduction to Information Retrieval Cambridge University Press; 2008.

12. Carroll HD, Kann MG, Sheetlin SL, Spouge JL: Threshold Average Precision (TAP-k): a measure of retrieval designed for bioinformatics. Bioinformatics 2010, 26(14):1708-1713.

13. Rinaldi F, Kaljurand K, Saetre R: Terminological resources for Text Mining over Biomedical Scientific Literature. Journal of Artificial Intelligence in Medicine 2011, 52(2):107-114.

14. Kaljurand K, Kappeler T, Schneider G: Using existing biomedical resources to detect and ground terms in biomedical literature. Proceedings of the 12th Conference on Artificial Intelligence in Medicine (AIME09) 2009, 225-234.

15. Rinaldi F, Kappeler T, Kaljurand K, Schneider G, Klenner M, Clematide S, Hess M, von Allmen JM, Parisot P, Romacker M, Vachon T: OntoGene in BioCreative II. Genome Biology 2008, 9(Suppl 2):S13 [http://genomebiology.com/2008/9/S2/ S13].

16. Rinaldi F, Schneider G, Kaljurand K, Clematide S, Vachon T, Romacker M: OntoGene in BioCreative II.5. IEEE/ACM Transactions on Computational Biology and Bioinformatics 2010, 7(3):472-480.

17. Tsuruoka Y, McNaught J, Tsujii J, Ananiadou S: Learning string similarity measures for gene/protein name dictionary look-up using logistic regression. Bioinformatics 2007, 23(20):2768-2774 [http://www.ncbi.nlm.nih.gov/pubmed/ 17698493].

18. Daumé H III: Notes on CG and LM-BFGS Optimization of Logistic Regression. 2004, [Paper available at http://hal3. name/docs/daume04cg-bfgs.pdf , implementation available at http://hal3.name/megam/1.

19. Dietterich TG: Approximate Statistical Tests for Comparing Supervised Classification Learning Algorithms. Neural Computation 1998, 10(7):1895-1923 [http://www.mitpressjournals.org/doi/abs/10.1162/089976698300017197].

20. Nadeau C, Bengio Y: Inference for the Generalization Error. Machine Learning 2003, 52(3):239-281.

21. Rinaldi F, Clematide S, Schneider G, Romacker M, Vachon T: ODIN: An Advanced Interface for the Curation of Biomedical Literature. Biocuration 2010, the Conference of the International Society for Biocuration and the 4th International Biocuration Conference 2010.

22. Arighi C, Roberts P, Agarwal S, Bhattacharya S, Cesareni G, Chatr-aryamontri A, Clematide S, Gaudet P, Giglio M, Harrow I, Huala E, Krallinger M, Leser U, Li D, Liu F, Lu Z, Maltais L, Okazaki N, Perfetto L, Rinaldi F, Saetre R, Salgado D, Srinivasan P, Thomas P, Toldo L, Hirschman L, Wu C: BioCreative III interactive task: an overview. BMC Bioinformatics 2011, 12(Suppl 8):S4 [http://www.biomedcentral.com/1471-2105/12/S8/S4].

23. Rinaldi F, Clematide S, Garten Y, Whirl-Carrillo M, Gong L, Hebert JM, Sangkuhl K, Thorn CF, Klein TE, Altman RB: Using ODIN for a PharmGKB re-validation experiment. Database: The Journal of Biological Databases and Curation 2012.

24. Rinaldi F, Clematide S, Hafner S: Ranking of CTD articles and interactions using the OntoGene pipeline. Proceedings of the 2012 BioCreative workshop, Washington DC, 04 April 2012 - 05 April 20122012.

25. Leaman $R$, Gonzalez G: BANNER: an executable survey of advances in biomedical named entity recognition. Pacific Symposium On Biocomputing Pacific Symposium On Biocomputing 2008, 13:652-663 [http://www.ncbi.nlm.nih.gov/ pubmed/18229723].

26. Aronson AR, Lang FM: An overview of MetaMap: historical perspective and recent advances. Journal of the American Medical Informatics Association 2010, 17(3):229-236 [http://www.ncbinlm.nih.gov/pubmed/20442139].

27. Tsai RTH, Hung HC, Dai HJ, Lin YW, Hsu WL: Exploiting likely-positive and unlabeled data to improve the identification of protein-protein interaction articles. BMC Bioinformatics 2008, 9(Suppl 1):S3 [http://www. pubmedcentral.nih.gov/articlerender.fcgi?artid=2259404n\&tool=pmcentrezn\&rendertype=abstract].

28. Giuliano C, Lavelli A, Romano L: Exploiting shallow linguistic information for relation extraction from biomedical literature. Proceedings of the Eleventh Conference of the European Chapter of the Association for Computational Linguistics EACL2006 2006, 18:5-7 [http://acl.ldc.upenn.edu/E/E06/E06-1051.pdf].

29. Bundschus M, Dejori M, Stetter M, Tresp V, Kriegel HP: Extraction of semantic biomedical relations from text using conditional random fields. BMC Bioinformatics 2008, 9:207 [http://www.pubmedcentral.nih.gov/articlerender.fcgi? artid=2386138n\&tool=pmcentrezn\&rendertype=abstract].

30. Miyao Y, Sagae K, Sætre R, Matsuzaki T, Tsujji J: Evaluating contributions of natural language parsers to proteinprotein interaction extraction. Bioinformatics 2009, 25(3):394-400 [http:/www.ncbi.nlm.nih.gov/pubmed/19073593].

doi:10.1186/2041-1480-3-S3-S5

Cite this article as: Clematide and Rinaldi: Ranking relations between diseases, drugs and genes for a curation task. Journal of Biomedical Semantics 2012 3(Suppl 3):S5. 